\title{
La Memoria Histórica: Camino de Reconocimiento de la Alteridad en el Conflicto Armado Colombiano
}

\section{The historical memory: road of recognition of the alterity in the colombian armed conflict}

\author{
Juan Carlos Gómez Quitian iD
}

Universidad Católica de Colombia

jcgomezq@ucatolica.edu.co

\section{Armando Rojas Claros}

Universidad Católica de Colombia

arojas@ucatolica.edu.co

\begin{abstract}
Resumen
El presente informe de investigación muestra cómo la memoria histórica puede convertirse en un camino educativo para el fortalecimiento de la alteridad en los estudiantes del curso de lógica y psicología de la Universidad Católica de Colombia, quienes han sido víctimas o espectadores del conflicto armado colombiano. Los participantes del estudio fueron los estudiantes de primer semestre de psicología, pertenecientes a la Universidad Católica de Colombia, de la asignatura Psicología y lógica; dos de los alumnos fueron víctimas directas, los demás han sido espectadores del conflicto armado colombiano. La información obtenida se sometió a un análisis de contenido de acuerdo con las categorías deductivas: alteridad, conflicto armado y memoria histórica.

Los resultados presentan la invisibilización de la persona, ya que han sido víctimas del impacto dentro del conflicto armado colombiano, con unas características propias del conflicto, el olvido y el silencio en contra de la misma humanidad por medio de la violencia que ha deshumanizado y desvirtualizado al ser, llegando a ser calificados como aquellos que son Nadie.

Por otra parte, la vulneración de los derechos humanos hacia las víctimas, por parte del estado y los grupos armados al margen de la ley, es indispensable para la memoria histórica como proceso educativo que lleva al reconocimiento de las personas, como miembros activos de la sociedad.
\end{abstract}

Palabras clave: conflicto armado, memoria, estudiante, reconocimiento.

\begin{abstract}
This research report shows how historic memory can be turned into an educational path for the strengthening of the otherness of the Catholic University of Colombia Logics and Psychology students who have been victims or observers of the armed conflict in Colombia. The field study was carried out with 15 students, men and women, from the first semester of Psychology at Catholic University of Colombia, registered in the Logics and Psychology subject, who have been observers of, direct victims of, or have knowledge about the Colombian armed conflict. The information gathered was subject to a content analysis according to the deductive categories otherness, armed conflict and historic memory. The results show that the process of invisibilization of the impact actually exists. The characteristics and consequences of the armed conflict that the victims have suffered, plus the oblivion they have been subject to through inhuman acts as silence, torture, violence, they have lost their families, cultures, towns, to be dubbed the Nobodies.
\end{abstract}


The violation of human rights towards the victims have been a process alongside historic memory that is an educational and formative path towards the recognition of otherness, from the narrative and testimony of the students, to raise awareness and create recognition of the other as person, with a face of their own, human, to accept him / her as an active part of the Colombian society

Keywords: armed conflict, historic memory, person, students, recognition and otherness.

Articulo: Recibido el 15 de septiembre de 2019 y aprobado el 16 de marzo de 2020

\section{Cómo citar este artículo:}

Gómez Quitián, J.C. \& Rojas- Claros, A. (2020). La Memoria Histórica: Camino de Reconocimiento de la Alteridad en el Conflicto Armado Colombiano. Reflexión Política 22(44), pp. 87-96. doi: 10.29375/01240781.3713

\section{Introducción}

El conflicto armado colombiano "ha perdurado por más de 50 años" (Jaramillo, 2015, p. 3), y su mayor protagonista ha sido la población civil al aportar más del $81 \%$ de los muertos, según cifras del Centro Nacional de Memoria Histórica, (2013, p. 23). Es así que el conflicto armado colombiano ha violado el Derecho Internacional Humanitario en el principio de distinción, que no ha sido una guerra de combatientes, del estado y de grupos al margen de la ley, sino que han apuntado sus fusiles a los campesinos, niños, mujeres, Lesbianas, Gays, Transexuales, Bisexuales e Intersexuales (LGTBI), y comunidades afro.

El conflicto armado colombiano tiene un detonante determinante, de acuerdo con el Centro Nacional de Memoria Histórica "La tierra está en el corazón del conflicto colombiano." (p. 49). Es así que el conflicto por la tierra es la principal causa de enfrentamiento entre grupos al margen de la ley y el Estado, lo que ha generado la ausencia de alteridad de cada persona y de comunidades involucradas en el conflicto, obteniendo la deshumanización en especial, "la población civil ha vivido una sucesión cotidiana de eventos de pequeña escala como asesinatos selectivos, desapariciones forzosas, masacres, secuestros, violencia sexual y minas antipersonales, entre otros." (Centro Nacional de Memoria Histórica, 2013, p. 19). Pueblos y municipios que no se conocían antes del conflicto armado se conocieron por la crueldad y sevicia con la cual el conflicto se ensañó "sobre los pueblos afrocolombianos e indígenas, se ensaña contra los opositores y disidentes, y afecta de manera particular a las mujeres, a los niños y niñas." (Centro Nacional de Memoria Histórica, 2013. p. 25).

Tales vulneraciones hacia algunas comunidades han tenido repercusiones fuertes, por ejemplo, el sufrimiento humano haciendo del conflicto un proceso deshumanizador perdiendo la sensibilidad por el otro.

La ordinariez de la vida, al ver el conflicto en dos momentos: el primero ver el conflicto armado como algo normal, natural de la vida y en segundo lugar el conflicto que irrumpe y transforma el orden natural de los ciudadanos, donde se concluye que el conflicto tiene un poder destructivo. (Galvis, 2014. p. 86)

\section{Diseño metodológico}

Los impactos que ha tenido la violencia en nuestro país pueden ser cuantificados en años, pérdidas económicas, y hasta número de muertos. Sin embargo, acercarse así a esta realidad no es suficiente para entender y aproximar el impacto que la violencia ha tenido en nuestro país, menos con estudiantes de primer semestre en la ciudad de Bogotá. Por esta razón, la presente investigación se trabajó desde un enfoque cualitativo, teniendo en cuenta que dicha metodologia permite dar cuenta de un proceso activo, sistemático y riguroso de indagación dirigida.

Adicionalmente, este enfoque permite un encuentro directo con la población, así mismo, "estudia la realidad en su contexto natural, tal y como sucede, intentando sacar sentido de, o interpretar los fenómenos de acuerdo con los significados que tienen para las personas implicadas" (Rodríguez, Gil, y García, 1996, p. 32). De esta manera, y tal como lo plantea la investigación cualitativa, es posible 
acercarnos a los acontecimientos, acciones, normas y valores desde la experiencia de las personas que están siendo estudiadas, de tal modo como si estas experiencias estuvieran pasando por primera vez, es decir, nada se da por sobreentendido, todo es un tema de investigación.

Dentro de este enfoque se trabajó la investigación descriptiva, la cual pretendió referir o narrar características y propiedades de un objeto, sujeto o situación específica, sin emplear juicios de valor y en procura de objetividad. En la investigación, esto permitió reconocer la urgencia del abordaje altérico del conflicto armado colombiano, y cómo en la realidad social que fue reconstruida, con la participación de los estudiantes, la memoria histórica resulta una estrategia generadora de una realidad dinámica.

El universo de estudio recogió un grupo de 21 estudiantes de la Universidad Católica de Colombia, estudiantes de la facultad de Psicología de primer semestre, y matriculados en el curso Lógica y psicología. Los criterios para la selección de los participantes fueron:

- Pertenecer a la facultad de psicología, y a la asignatura de Lógica y psicología.

- Pertenecer al grupo poblacional al que se le realizó el primer cuestionario.

- Interés en el tema de investigación y motivación de querer profundizar en él voluntariamente.

- Ser espectadores del conflicto, directa o indirectamente.

- Presentar algún grado de cercanía frente a una víctima o a su testimonio.

Teniendo en cuenta los criterios anteriores, se seleccionaron 15 estudiantes de los cuales 13 eran espectadores o víctimas indirectas $y$ 2 resultaron ser víctimas directas del conflicto armado colombiano. En cuanto al género, 12 eran mujeres y 3 hombres. Por otro lado, las edades de los participantes oscilaban entre los 18 y los 37 años.

\section{El reconocimiento de los otros}

Del encuentro y el reconocimiento de la alteridad nace un nuevo humanismo, por medio de la responsabilidad y el compromiso, y en la relación de alteridad se presenta la vulnerabilidad, por lo tanto, es crear una identidad nueva, donde se manifieste la relación que afecta, hace sufrir, luchar y estar expuestos a las heridas de la sociedad, de manera que es "poner fin a todas las violaciones del Derecho Internacional Humanitario y buscar y enjuiciar a las personas que hayan cometido violaciones graves del DIH en conflictos armados internacionales" (Salmon, 2012, p. 111), de modo que la alteridad se hace como una forma de generar espacios y encuentros para una mejor relación de humanidad.

El reconocimiento de la alteridad no sucede en un tiempo y espacio determinado, sino que es el encuentro con el otro por medio de "la epifanía del rostro" (Levinas, 1974, p. 59), que es la revelación del otro, donde se desnuda su vida por medio de relaciones personales, laborales y comunitarias, y se desvela hacia un reconocimiento de la alteridad, donde irrumpe la llegada del otro e "inicia a entrar en mi mundo, me desubica, se me impone, queriendo decir es el compromiso por el otro ¡no me mates!”, "el otro nos confronta fuera del contexto y así viene con un rostro desnudo, la desnudez del rostro es un despojamiento sin ornamentos culturales, una absolutización.”, "es el conocimiento del cara a cara con el otro que interpela mi mundo, me invade con el fin de conocer la alteridad, el otro que existe en mi mundo, que es tan cercano como tan desconocido." (Levinas, 1974, p. 59).

La relación del pueblo colombiano de vivir en medio del fuego cruzado por enfrentamientos entre las fuerzas armadas, las guerrillas y los paramilitares, la reiteración de las agresiones, las constantes amenazas y los asesinatos diarios, han roto la alteridad, sin importar el tú, solamente importa el yo, y como lo llama Levinas (1974) en su libro Humanismo del otro hombre la "nonindifference", que consiste en que no hay una relación, pero tampoco hay indolencia. Es así que Colombia ha tenido fallos en contra por la vulneración de los derechos humanos, (Corte interamericana de derechos humanos, 2018), es el caso vereda la esperanza us. Colombia, en el cual indemnizan a la señora Nelly Soto de Castaño y a Verónica Giraldo Soto, madre e hija, respectivamente, por la ejecución extrajudicial del señor Javier Giraldo.

Otro fallo de la Corte en contra de Colombia es el caso Omeara Carrascal us. Colombia, en el cual se fundamenta que por manos de los grupos paramilitares, que la Comisión expresó que 
el caso se relaciona con el atentado sufrido por el señor Noel Emiro Omeara Carrascal (en adelante también "Noel Emiro Omeara" o "señor Omeara Carrascal”) el 28 de enero de 1994 y su posterior muerte; la desaparición y ejecución de Manuel Guillermo Omeara Miraval (en adelante también "Manuel Guillermo Omeara" o "señor Omeara Miraval”), hijo del primero, desde el 27 de agosto al 23 de septiembre de 1994, y el atentado y posterior muerte del señor Héctor Álvarez Sánchez (en adelante también "Héctor Álvarez" o "señor Álvarez Sánchez"), suegro del segundo, el 21 de octubre de 1994. (Corte Interamericana de Derechos Humanos, 2018)

La invitación de Levinas (1974) en su libro Humanismo del otro hombre es la sensibilidad por el otro, que se ve reflejada en el rostro, en la comunicación de la palabra y en el discurso, el cual permite establecer una responsabilidad del yo hacia el otro en un clima de encuentro de cara a cara, y en la cual se inicia una relación que trasciende por medio del rostro que transmite cultura, sentimiento, valores, experiencia de vida, como lo dice Levinas (2002) "El rostro, su revelación es palabra. Sólo la relación con el otro, nos conduce hacia una relación totalmente diferente de la experiencia en el sentido sensible del término" (p. 217). Es decir, la relación con el otro saca de la cotidianidad al ser para tener una relación directa y responsable con el otro por medio del reconocimiento.

En este sentido, el reconocimiento altérico y el encuentro con el otro, sabiendo que él es quien ayuda en la autoformación y auto liderazgo, cuya característica principal es ser un impulso que lleva, como menciona Castro (2001), está "fuera de sí, hacia lo otro que es uno mismo" (p. 34) para encontrarse con la realidad de quien es. De esta manera, el otro interpela la propia vida, la existencia, haciendo que, de alguna manera, se reconozca la importancia del otro en la reconstrucción de la historia, evidenciando la necesidad de la apertura hacia ese otro ante la falta de solidaridad que se ha vivido y suscitando el compromiso y respeto que debe darse a la vida de ese ser.

Levinas (2002) interpela al mencionar que, "en la expresión: un ser se presenta a sí mismo, sin necesidad de que éste pronuncie palabra alguna"; hoy, en los periódicos, noticieros y entrevistas, la misma sociedad se refiere al otro como guerrilleros, terroristas, víctimas, los presuntos ladrones, jaladores, expendedores, basuqueros, drogadictos, gamines, desechables, gays, lesbianas y transexuales. Es la deshumanización del otro, de su rostro, es un adjetivo despectivo para referirse al ser como persona, y esto conlleva a perder la naturaleza por la relación humana. Como plantea Guesada (2011) "La alteridad es relación y lenguaje: se le da al Yo por la palabra; es subjetividad: forman una sociedad en la responsabilidad del Yo para con el Otro; es justicia y verdad, libertad" (p.180). El pensamiento de Levinas 2011, en Colombia es una utopía, o un reto que en la realidad se hace necesaria para establecer relaciones y crear un lenguaje en pos del humanismo, pues, según Navarro (2007), "la significación del rostro es la de un compromiso ético anterior a toda etnia, cultura, identidad e ideologia” (p.183), es el compromiso ético ante el otro en dos momentos, "el primero en el reconocimiento altérico, y el segundo la relación de alteridad que parte de la capacidad del rostro" (Navarro, 2007, p.183) de leer al otro como presencia de humanidad y divinidad.

La significación del rostro sólo puede ser ética y por ello, los términos miseria, pobreza y hambre que aparecen una y otra vez en la argumentación Levinasiana para hacer referencia a la misma, no describen físicamente al otro, sino concentran la significación a través de la cual su presencia se impone éticamente. Lo que simplemente quiere decir que la miseria, el hambre y la pobreza del otro, no sólo invocan al yo, sino que también lo interrogan y exigen una respuesta. (Navarro, 2007. p.184)

Hoy nuestra sociedad es líquida, sin horizonte, es el rechazo a comprometerse con y por el otro, es decir, es una sociedad desechable que al otro no le interesa, no es reconocido, y se han creado relaciones o comunidades frágiles, sin una razón de ser, es el miedo al amor altérico, comprometido y responsable para reconocer en el otro la importancia de construir la historia personal y colectiva. Zygmunt Bauman (2003) se encarga bien de recalcar este aspecto, "que ni son dilemas locales ni pueden resolverse de manera local, pero la dificultad es que la humanidad no dispone todavía de otra organización institucional sólida que no sea la que ofrece la modernidad, la que justamente, día a día, transita su desintegración." (p.160).

Una desintegración en pos de la destrucción y ruptura de sistemas bien conformados, como lo son la familia y todas las relaciones que están 
en crecimiento mutuo en favor de reconocer y comprometerse por el reconocimiento de la alteridad. En esta perspectiva, conformar una familia, tener hijos, son verdaderos obstáculos para la libertad, y en especial para el reconocimiento del otro. De acuerdo con Navarro (2007) "sólo el yo es responsable del otro y no a la inversa” (p.187).

Un ejemplo claro de la vulneración de la alteridad fue la violación al "derecho a la vida, a la libertad y a la seguridad de la persona" (Asamblea General de la ONU, artículo 3). La forma en la cual el terror recorrió las calles de la Comuna 13 de Medellín a finales de la década de los 90, tras el asentamiento de milicias urbanas de las Fuerzas Armadas Revolucionarias de Colombia (FARC), el Ejército de Liberación Nacional (ELN) y Comandos Armados del Pueblo, fue testigo en múltiples ocasiones de la forma en la que aniquilaron la vida de varias personas.

\section{La memoria histórica: camino de reconocimiento}

La memoria histórica cumple la función de "la recuperación socioafectiva de personas y comunidades" (Gaborit, 2006, p. 671), transportando el pasado al presente de manera consciente, y así, la experiencia que han vivido las víctimas y victimarios no sea ajena a aquellos que han sido espectadores directos o indirectos de las situaciones objeto de recuerdo; como lo afirma la ley 1448 de 2011 en su artículo 149 de la ley de víctimas y restitución de tierras, la memoria histórica se usa como un instrumento social "con el fin de no repetir hechos victimizantes" y el no olvido del pasado, para que el mismo país no se vuelva cómplice de los hechos que lo han marcado, que han generado terror y horror, y así evitar el silencio, y evitar la participación en el anonimato de la mayoría de las víctimas que ha arrojado y dejado a la deriva el conflicto armado colombiano.

La importancia del levantamiento de memoria histórica en el ámbito educativo establece una relación directa entre la academia, especialmente entre los estudiantes con la historia, específicamente en el capítulo del conflicto armado colombiano entre las víctimas y victimarios, para que no exista un memoricidio, sino todo lo contrario, generar el conocimiento de los hechos ocurridos y garantizar la no repetición de los mismos, como lo mencionan Silvera Sarmiento y Archila Guío (2018), en su libro Valores para una formación en Derechos Humanos, "Es así como la amistad y las virtudes del ser se consolidan como una herramienta de mediación pedagógica para la transformación de la práctica de los derechos humanos en contextos de paz y reconciliación.” (p. 20). En pos de una reconciliación y sanación de las barbaries que se han cometido en el conflicto armado, teniendo en cuenta que el pasado permanece en la memoria, y la historia sigue su camino.

En este sentido, los estudiantes del programa de Psicología en su curso de Lógica y psicología plantearon que hacer ejercicios de memoria histórica favorecen los procesos de sanación personal para afrontar un momento como el que políticamente se ha denominado postconflicto, (Acevedo y Rojas 2016) es “el proceso de paz es el conjunto de fases de negociación y de mediación, que permite llegar a un acuerdo entre las partes que se encuentran en conflicto" (p.41) como lo afirma una de las víctimas directas:

Bueno, hasta el momento nunca he hecho un tipo de memoria histórica excepto con los docentes. Pienso que si le ayuda a uno para hablar con alguien tranquilamente y de esta manera soltar toda esa frustración que uno siente y tristeza de la situación que vivió y que lo entienden a uno. Se siente uno como se quita un peso de encima porque pues uno sabe que uno con todo mundo no puede hablar del tema, porque pues muchas personas pueden ser que lo juzguen, otras personas dirán que es mentira, o sea, pero me siento tranquila.

Tal y como narra la víctima, la memoria histórica pasa por un diálogo desde lo real, en la relación del tú a tú que la alteridad favorece, y que permite expresar el sentimiento que ha vivido, las huellas que han quedado, el pasado que no se puede borrar, y la historia que pasa entre las generaciones que no puede ser ignorada, ya que "Colombia se debate en la disyuntiva de ahogarse en el mar de la violencia o salir a flote" (Silvera y Archila, 2015, $\mathrm{p}$.43). En el espacio del reconocimiento, que tiene como uno de los mediadores el arte de escuchar para garantizar la dignidad que han perdido algunas personas a causa del conflicto armado colombiano por el anonimato, el silencio y el olvido que nace del memoricidio que vive la misma sociedad, por lo tanto se hace indispensable el realizar ejercicios de memoria histórica. 
Unido a lo dicho, los grupos focales que se abordaron desde la memoria histórica ratifican la validez de la misma como espacio pedagógico, en cuanto a los ejercicios y a la confrontación entre los estudiantes de sus creencias y conocimiento han generado que "la emergencia de las memorias de resistencia y del reto de hacer memoria histórica como instrumento de paz, son leídos como signos de un nuevo tiempo y oportunidad de otro futuro" (Centro de Memoria, Paz y Reconciliación, 2014, pág. 8), para realizar el siguiente paso que es el ver, leer y escuchar la memoria histórica de aquello que ha dejado el conflicto armado colombiano, dejando claro que la historia real queda grabada en el ser del otro y no en los libros.

Así, la memoria histórica se presenta para los estudiantes universitarios como un tema importante de actualidad; vivenciarlo desde la parte educativa es percibido como un ejercicio acertado en espacio y tiempo, porque ellos han podido realizar ejercicios acerca de los temas de conflicto armado colombiano, memoria histórica y alteridad, llegando a entender que la sociedad ha estado largamente fracturada por el acontecer de un conflicto, con objetivos claros, el poder político y el dominio de las tierras.

La importancia de meter lo civil en la definición está en superar la idea de una guerra de aparatos que se autodeterminan. Además, indican que, en definitiva, la lógica de las operaciones armadas ha sido someter a la población civil: en la violencia estatal o en la insurgencia y contrainsurgencia, en las guerras por poder o por recursos, el objetivo ha sido el sometimiento de la población o transformarla en parte activa en la lucha contra el enemigo. (Centro de Memoria, Paz y Reconciliación, 2014, p. 31)

Para someter y subordinar a ciertos sectores de la población se hace uso de la agresión por medio de las masacres, por ejemplo, el caso del arrasamiento paramilitar en el Sur de Bolivar en la segunda mitad de la década del noventa; el caso en Segovia, Antioquia, el 11 de noviembre de 1988, a manos de los paramilitares; en Bojayá, el 02 de mayo de 2002, crimen cometido por la guerrilla de las Farc; la masacre del Salado entre el 16 y 22 de febrero del 2000, perpetrada por los paramilitares; las masacres sucedidas en san Carlos, Antioquia, generadas por todos los actores armados, siendo víctimas de masacres, asesinatos selectivos de líderes cívicos, 156 desapariciones forzadas, violencia sexual contra las mujeres, tomas al pueblo y extorsión; en Bahía Portete, Alta Guajira, entre el 18 y 20 de abril de 2004, los paramilitares llegaron con lista en mano e incendiaron casas, torturaron y asesinaron a varias personas.

\section{La falta de intersubjetividad que dio pie a la barbarie}

La definición de conflicto armado interno se encuentra en el Protocolo II adicional a los acuerdos de Ginebra, que fue ratificado por Colombia en 1994. Según el Protocolo II de los mencionados convenios, se habla de "conflicto armado de carácter no internacional” (Comité Internacional de la Cruz Roja, 2008) cuando un Estado es confrontado por una o varias fuerzas armadas irregulares.

En el artículo 1 se definen como:

Conflictos armados [aquellos] que se desarrollen en el territorio de una Alta Parte contratante [es decir Estado firmante] entre sus fuerzas armadas y fuerzas armadas disidentes que, bajo la dirección de un mando responsable, ejerzan sobre una parte de dicho territorio un control tal que les permita realizar operaciones militares sostenidas y concertadas (...). (p. 1)

El conflicto armado es la guerra que forjan diversos grupos al margen de la ley, (FARC, ELN, Ejército popular de liberación (EPL), Alianza Democrática M 19 (M-19), y paramilitares) contra el Estado para alcanzar sus intereses políticos, militares, económicos, culturales y territoriales con el fin de tomar el dominio del Estado y de la población civil, quienes son los que sufren el horror del conflicto.

La ley 1448 de 2011 articulo 3, se refiere a las víctimas de la siguiente manera

Víctimas: Se consideran víctimas, para los efectos de esta ley, aquellas personas que individual o colectivamente hayan sufrido un daño por hechos ocurridos a partir del $1^{\circ}$ de enero de 1985 , como consecuencia de infracciones al Derecho Internacional Humanitario o de violaciones graves y manifiestas a las normas internacionales de Derechos Humanos, ocurridas con ocasión del conflicto armado interno.

Además, porque son vulnerados sus derechos y son revictimizadas por el dominio de la misma sociedad. 
Según el Registro Único de Víctimas (RUV), desde 1985 hasta el 20 de febrero de 2020 se reportaron 8.970.712 civiles víctimas fatales del conflicto armado, discriminándolos de la siguiente manera:

Hombres: 4.469.709

Mujeres: 4.495 .222

LGTBI: 4.425

No informa: 310

Sin embargo, este balance es parcial debido a que el marco legal solo reconoce a las víctimas a partir del primero de enero de 1985, lo que excluye a 11.238 víctimas documentadas en la base de datos del Grupo de Memoria Histórica (GMH).

Así mismo, es importante señalar que en el RUV no están incluidos los combatientes muertos en las acciones bélicas. De acuerdo con la investigación del GMH al compendiar estas cifras, es posible afirmar que el conflicto armado colombiano ha provocado aproximadamente 220.000 muertos.

De estas muertes, el $81,5 \%$ corresponde a civiles y el 18,5\% a combatientes; es decir que aproximadamente ocho de cada diez muertos han sido civiles, y que, por tanto, son ellos - personas no combatientes -, según el Derecho Internacional Humanitario, los más afectados por la violencia (Centro Nacional de Memoria Histórica. 2013, pág. 32).

$\mathrm{El}$ accionar de los grupos al margen de la ley, guerrillas, paramilitares y las fuerzas del Estado, dan inicio a un conflicto armado el cual ha violado los derechos humanos y el derecho internacional humanitario.

Los paramilitares instalaron un retén en la vía Canutal-Flor del Monte, y allí detuvieron a Emiro Castillo Castilla, quien se transportaba en una moto; lo hicieron bajar de ésta, lo amarraron de las manos y lo degollaron. La moto fue quemada. (Miembros del Grupo de Memoria Histórica, 2009, p. 29)

Dichas violaciones son cometidas en contra de cada persona y/o comunidad, vulnerando sus derechos, este es el caso de San Carlos, Antioquia, "hubo por lo menos 33 masacres, se registraron 156 desapariciones forzadas y 78 personas fueron víctimas de las minas antipersonal" (Grupo de Memoria Histórica, 2011, p. 28), violentado su condición física, social, cultural y religiosa, teniendo como consecuencia la inestabilidad del individuo, porque han atropellado su identidad como persona, el placer por la dominación al violentar y el poder traducido en la persona, las tierras, dinero que el otro haga, como afirma Arendt (2012) "instinto de poder" (p.50) y, de esta manera, realice lo que yo quiero y le impongo vulnerando los derechos humanos del otro.

El país ha tenido toda clase de tragedias, toda clase de dolor. No hay familia en Colombia que esté exenta de que alguno de sus integrantes haya sido erradicado del mundo de los vivos por la maldita violencia. Colombia está corrompida por las violencias. Desde la Patria Boba, hasta todo lo largo del siglo XIX, con los enfrentamientos fratricidas en las guerras civiles, con una calma chicha entre 1903 y 1930, cuando empieza la violencia política y luego el recrudecimiento, a partir de 1948. (Centro de Memoria, Paz y Reconciliación, 2014. p. 48)

El conflicto armado colombiano sometió a las comunidades afrodescendientes y a la comunidad LGBTI, a la desaparición forzada, ya que los paramilitares querían tener el control de la zona, ellos lo llamaban un régimen cultural, es así que "La violencia sexual registrada corresponde en su mayoría a casos perpetrados por los paramilitares (15) frente a un caso en que se identifica a las guerrillas como autoras del crimen" (Centro Nacional de Memoria Histórica, 2019, p. 114), tanto así que la autoridad que se encontraba en algunos pueblos o municipios era ellos mismos; Galvis (2014) comparte las acciones que tomaban los miembros de las comunidades.

El conflicto armado colombiano vulnera las reglas del derecho internacional humanitario; tiene unas características que han deshumanizado a la población civil y ha traído consecuencias, por ejemplo, como lo menciona el Centro Nacional de Memoria Histórica (2017) "La permanencia de estos grupos armados con sus respectivas reconfiguraciones ha puesto en evidencia la implementación de acciones de reclutamiento de niños, niñas y adolescentes bajo la modalidad de persuasión." (p. 274), como se narra en el libro una guerra sin edad, del Centro Nacional de Memoria histórica (2017).

Uno de niño quiere jugar con sus juguetes, su muñeca. Mi niñez fue andar en uniforme, mijuguete un fusil. El miedo que de pronto me mataran. Era 
que en cualquier momento me mataran y que en cualquier momento el comandante lo obligara a uno a estar con él. Ese era mi principal miedo. Que lo obligaban a uno a tener relaciones con ellos. (CNMH, joven desvinculada de las FARC-EP, entrevista facilitada por el proyecto Las Voces de los Niños, Niñas y Adolescentes, Villavicencio, 10 de octubre de 2013, p. 516)

La muerte entró en el proyecto de vida de las comunidades, así lo afirma Galvis (2014) "la muerte como posibilidad cercana" (p. 108), porque la vida se encuentra con la muerte cara a cara, y es así que se inician décadas de escuchar muertos y "todos los días se veían muertos" (Centro Nacional de Memoria Histórica, 2015, p. 97), es decir, el conflicto se rutinizó, empezó hacer parte del proyecto de vida de las comunidades, esto es un claro ejemplo para realizar el levantamiento de memoria histórica en los estudiantes frente al concepto de alteridad en un conflicto que en "Colombia ha padecido una crisis humanitaria de proporciones indecibles a manos del conflicto armado" (Centro Nacional de Memoria Histórica, 2015, p. 23).

Colombia ha vivido las últimas décadas en luto permanente. Masacres y otras formas de violencia colectiva con diversas magnitudes, intencionalidades y secuelas han ensangrentado la geografía nacional. Colombia ha vivido no sólo una guerra de combates, sino también una guerra de masacres. Sin embargo, la respuesta de la sociedad no ha sido tanto el estupor o el rechazo, sino la rutinización y el olvido. (Miembros del equipo de Memoria Histórica, 2008, p.11)

El conflicto tocó la vida cotidiana de las comunidades de tal forma que este hizo parte del proyecto de vida de las mismas, así lo argumenta (Morales, 2017).

Las víctimas del conflicto armado colombiano han sobrepasado lastimosamente récords, empezando por las cifras en desplazamiento forzado, como lo menciona Lorenzo Morales (2017), para el 2016 "Colombia es el país con más migración interna después de Siria” (p. 5).

La violación de la alteridad hacia la infancia se vio vulnerada en

aquellos niños y jóvenes que han sido reclutados para ser ingresados a las filas de grupos al margen de la ley, trayendo consigo violaciones a normas internacionales como la Convención sobre
Derechos del Niño adoptada por Asamblea General de las Naciones Unidas el 20 de noviembre de 1989. (Gónzalez y Carrasquilla, 2017, p. 59)

ElRUV hasta el 20 de febrero de 2020 reportó 8.194 casos de menores reclutados en el conflicto armado colombiano; un claro ejemplo se evidencia en el reclutamiento de menores de edad; esto una problemática que aún se mantiene en el cuarto periodo del conflicto armado, la deshumanización que genera la guerra en un niño no solo afecta al menor, sino a su entorno, un evidente ejemplo de lo anterior es "la muerte de 8 niños a manos del ejército en un bombardeo autorizado a disidencias de guerrillas" (El espectador, 2019, p. 1). Esta noticia se dio a conocer tiempo después del hecho, justo cuando algunos senadores le mostraron la verdad al país, verdad que las fuerzas armadas querían ocultar; por este hecho, una madre solo podrá dar cristiana sepultura a una sola extremidad inferior de su hija, otras familias no podrán tener la capacidad de enterrar un cuerpo porque este dejó de existir.

Del mismo modo, se plantea la necesidad de reconocer que los vejámenes acogidos en el desarrollo del conflicto también aludieron a ataques en conjunto, desde asesinatos grupales hasta masacres en las que se exterminaba con las vivencias de pueblos enteros, una violación del Artículo 5 "Nadie será sometido a torturas ni apenas o tratos crueles, inhumanos o degradantes" (Asamblea General de la ONU, 1948), y exponiendo la atrocidad que caracterizó estos sucesos, por ejemplo, la utilización, por parte de los paramilitares, de perros pitbull y jaguares para atemorizar y agredir a quienes señalaban de colaboradores de la guerrilla, o la constitución de hornos crematorios por parte del Bloque Catatumbo, a los que fueron llevados líderes, defensores de los derechos humanos y simpatizantes de izquierda tras su asesinato, lo que constituye una violación al Artículo 19 "todo individuo tiene derecho a la libertad de expresión".

Afrontar desde la memoria histórica este hecho, permitió en los estudiantes exponer incluso el dolor patriótico, y el asombro frente a la crudeza de la guerra en un país sin medios económicos, porque según ellos, "la pobreza científica e industrial se convierte en una creatividad macabra y que olvida al otro totalmente, lo minimiza y lo cosifica". Y como afirma el Centro Nacional de Memoria Histórica (2013), “estas muertes ocurrieron de 
manera cotidiana, selectiva, silenciosa, en partes muy alejadas de los centros urbanos, y por tanto, han pasado inadvertidas para la mayoría de la sociedad. Y segundo, que estuvieron acompañadas de crueldad y terror." (p. 35)

\section{Conclusiones}

En el plano de la discusión de la Memoria histórica, el conflicto armado y la alteridad, frente a temas como la filosofía, la política y la historia, juega un papel trascendental la educación, al escuchar la voz de las víctimas y la memoria histórica en el reconocimiento de la alteridad, y así conocer la verdad de los hechos por medio del equilibrio entre el pasado y el futuro, justicia y verdad, perdón y reconciliación, víctimas y victimarios, conflicto y paz.

La no repetición de los hechos victimizantes que se han desarrollado a lo largo de la historia del conflicto armado colombiano es una tarea social, política y educativa que debe ser asumida como una responsabilidad de todos, pues la vida es valiosa para trabajarla conjuntamente desde la ética del cuidado, que debe primar por encima de una ética marcada por la ley. Todo esto en el plano de lo ideal; pero adentrándose en lo real se aprecia que han sido muchos los argumentos que han estado presentes para atentar contra los derechos humanos, como por ejemplo los ideales y objetivos de la guerrilla por alcanzar el poder político y militar, los grupos paramilitares legales o ilegales, y las fuerzas armadas del Estado por la lucha, defensa y garantía de los derechos humanos.

Los victimarios en nombre de su creencia han agudizado el conflicto armado, las víctimas y los espectadores conscientes o inconscientes han aportado al conflicto con la impotencia o con su indiferencia. En fin, el conflicto armado nos permea a todos, en su fondo, en su forma o en sus secuelas y de ahí la importancia de generar procesos educativos y restitutivos que estén orientados a la realización de ejercicios de memoria histórica que vayan de la negación de la alteridad a la restitución de la misma.

La memoria histórica es una estrategia educativa que busca el reconocimiento de la alteridad. Los procesos pedagógicos orientados a este propósito contribuyen con quienes han sobrevivido y son víctimas directas del conflicto armado colombiano. Los ejercicios de memoria histórica nos permiten un recorrido por los diversos momentos que se viven en el conflicto armado en el antes, el durante y el después de los hechos que cada víctima ha tenido que enfrentar. El objetivo es lograr que estos hechos no se queden en el olvido $\mathrm{y}$ nos ayuden a hacer conciencia de su nefasta repetición.

Las grandes mayorías de las sociedades latinoamericanas, que poseen una historia larga de represión y guerra, tienen necesidad de acceder a esa memoria como paso indispensable para obtener siquiera un módico de salud mental e ir configurando su identidad personal y colectiva.

(Gaborit, 2006, p.10)

De acuerdo con lo anterior, todas las huellas y cicatrices que llevan, portan y cargan las víctimas a partir de sus experiencias en primera persona, como consecuencia del conflicto armado colombiano, en el cual no tenían interés, tienen en la práctica de la memoria histórica un salvamento para ir sanando sus heridas.

\section{Referencias}

Acevedo Suárez, A., \& Rojas Castillo, Z. M. (2016). Generalidades del conflicto, los procesos de paz y el posconflicto. Revista de la facultad de derecho y ciencias políticas, 33 - 45.

Arendt, H. (2012). Sobre la violencia. Madrid: Santillana Ediciones Generales, S. L. Obtenido de http:// bello.cat/Sobre\%20la\%20violencia-H.\%20Arendt.pdf

Asamblea General de la ONU. (1948). Declaración Universal de los Derechos Humanos. París. Obtenido de https://www.un.org/es/universal-declaration-human-rights/

Bauman, Z. (2003). Modernidad liquida. Buenos Aires: Fondo de cultura económica.

Castro, R. (2001). Levinas y el humanismo del rostro. Revista electrónica Diálogos educativos, I(2). Obtenido de file:///C:/Users/Usuario\%2022/ Downloads/Dialnet-LevinasYElHumanismoDelRostro-2095845.pdf

Centro de Memoria, Paz y Reconciliación. (2014). Rompecabezas de la memoria ¿Aportes a una comisión de la verdad? Bogotá: Comité de Memoria Paz y Reconciliación.

Centro Nacional de Memoria Histórica. (2013). ¡Basta ya! Colombia: Memorias de guerra y dignidad. Resumen. Bogotá: Imprenta Colombia. Obtenido de http://www.centrodememoriahistorica.gov.co/descargas/informes2013/bastaYa/resumen-ejecutivo-basta-ya. pdf 
Centro Nacional de Memoria Histórica. (2015). Limpieza social. Una violencia mal nombrada. Bogotá: Centro Nacional de Memoria Histórica.

Centro Nacional de Memoria Histórica. (2017). Una guerra sin edad. Informe nacional de reclutamiento $y$ utilización de niños, niñas y adolescentes en el conflicto armado colombiano. Bogotá. Obtenido de http://www.centrodememoriahistorica.gov.co/descargas/informes2018/una_guerra-sin-edad.pdf

Centro Nacional de Memoria Histórica. (2017, a). Una guerra sin edad, Informe nacional de reclutamiento y utilización de niños, niñas y adolescentes en el conflicto armado colombiano. Bogotá: Centro Nacional de Memoria Histórica.

Centro Nacional de Memoria Histórica. (2019). Ser marica en medio del conflicto armado. Bogotá: Centro Nacional de Memoria Histórica.

Comité Internacional de la Cruz Roja. (2008). Cuál es la definición de "conflicto armado" según el derecho internacional humanitario. Ginebra. Obtenido de https://www.icrc.org/spa/resources/documents/article/other/armed-conflict-article-170308.htm

Corte interamericana de derechos humanos. (2018). Sentencia de 21 de noviembre de 2018. Obtenido de https://www.corteidh.or.cr/docs/casos/articulos/seriec 367 esp.pdf

El espectador. (6 de Noviembre de 2019). Judicial. El espectador, pág. 1 .

El Heraldo. (18 de Febrero de 2015). Las teorías del origen del conflicto armado en Colombia. El heraldo.

Gaborit, M. (2006). Memoria histórica: Relato desde las víctimas. Pensamiento Psicológico, II(6), 7-20. Obtenido de https://mail.google.com/mail/u/1/\#sent/ p4?projector $=1$

Gaborit, M. (2006, b). Memoria histórica: revertir la historia desde las víctimas. Estudios centroamericanos (61), 663 - 684.

Galvis, P. (2014). Narrativas de vida, dolor y utopías Jóvenes y conflicto en Colombia. Bogotá: Universidad de la Salle.

Gómez, P. (2012). Los muertos no hablan. Edición Bojayá, una década (2002 -2012). Medellín: Nuevo Milenio. Obtenido de https://www.traficantes.net/ sites/default/files/pdfs/los-muertos-no-hablan.pdf

González Ortiz, J. E., \& Carrasquilla Baza, D. (2017). Niños, niñas y adolescentes ¿víctimas o victimarios del conflicto armado en Colombia? Justicia Juris, 13(1), 56 - 62 .

Grupo de Memoria Histórica. (2011). San Carlos: Memoria del éxodo en la guerra. Bogotá: Taurus.

Grupo de Memoria histórica y Universidad British Columbia. (2013). Recordar y narrar el conflicto. Bogotá: Imprenta Nacional de Colombia.

Jaramillo, V. (2015). Un estudio sobre la internacionalización del Conflicto Armado en Colombia y su búsqueda por encontrar la paz duradera. Revista digital EJIL - EAFIT Journal of International Law, 6(2), 6 - 33.

Levinas, E. (1974). Humanismo del otro hombre. México: Siglo XXI Editores.

Levinas, E. (2002). Totalidad e Infinito. Salamanca: Sigueme. Obtenido de https://escuelacriticavaldiviana.files.wordpress.com/2012/06/levinas-1961-totalidad-e-infinito_ocr.pdf

Miembros del equipo de Memoria Histórica. (2008). Trujillo: una tragedia que no cesa. Bogotá: Planeta.

Miembros del Grupo de Memoria Histórica. (2009). La masacre de el Salado: Esa guerra no era nuestra. Bogotá: Taurus.

Montero, J. (2008). Memoria, olvido y perdón. La búsqueda del justo medio en la ley de justicia y paz. Bogotá: Ediciones Grancolombianas.

Morales, L. (2017). La paz y la protección ambiental en Colombia: Propuestas para un desarrollo rural sostenible. El diálogo, Bogotá.

Navarro, O. (2007). El "rostro» del otro: Una lectura de la ética alteridad de Emmanuel Lévinas. Revista Internacional de Filosofia, XIII, 177-194. Obtenido de file:///C:/Users/Usuario\%20 22 / Downloads / Dialnet-ElRostroDelOtro-2863805.pdf

Quesada, B. T. (2011). Aproximación al concepto de "Alteridad" en Levinas. Propedéutica de una nueva Ética como filosofía primera. Investigaciones fenomenológicas, III. Obtenido de file:///C:/Users/Usuario\%2022/Downloads / Dialnet-AproximacionAlConceptoDeAlteridadEnLevinas-4846508.pdf

Rodríguez Gómez, G., Gil Flores, J., \& García Jiménez, E. (Rodriguez, R., Gil , J., \& Garcia, E. de 1996). Metodología de la investigación cualitativa. Granada: Aljibe. Obtenido de http://www.catedranaranja.com.ar/taller5/notas_T5/metodologia_investig_cap.3.pdf

Salmón, E. (2012). Introducción al derecho internacional humanitario. Lima: Instituto de democracia y derechos humanos.

Silvera Sarmiento, A., \& Archila Guio, J. E. (2015). Victimología y valores humanos. Bogotá: Grupo Editorial Ibáñez.

Silvera Sarmiento, A., \& Archila Guio, J. E. (2018). Valores para una formación en Derechos Humanos. Bogotá: Grupo Editorial Ibáñez. 